\title{
WORK FORCE How we adapted the T\&O inpatient service during the COVID-19 pandemic with physician associates to support the orthopaedic team
}

\author{
Authors: Roz Tucker, ${ }^{A}$ Sverrir Kristinsson, ${ }^{B}$ Divya Benny, ${ }^{A}$ Zainab Khan, ${ }^{A}$ Tofunmi Oni ${ }^{C}$ and Andrew Smith ${ }^{D}$
}

\section{Introduction}

In March 2020, due to the COVID-19 pandemic, there were increasing demands on medical and intensive care departments in the UK. Medical staff from surgical departments were redeployed. The aim of this study was to determine whether the department was able to maintain standards with the use of the physician associate / medical doctor (PA/MD) model of care.

\section{Methods}

A mix of questionnaires and audit data was collected prospectively and compared with pre-COVID and the general surgical team which did not have PAs.

Results

Sixty-five per cent of responses indicated an improvement compared with pre-COVID conditions and $35 \%$ indicated care was the same. The electronic discharge notification audit showed an $89 \%$ completion rate for orthopaedics compared with $73 \%$ for general surgery. Venous thromboembolism assessment compliance was better compared with general surgery.

Conclusion

Overall, the study supports the hypothesis that a PA/MD model of care is non-inferior to a MD-only model of care and was effective.

KEYWORDS: physician associate, COVID-19, trauma and orthopaedics, adapted, pandemic

DOI: $10.7861 /$ fhj.2020-0206

\section{Introduction}

The UK went into lockdown on 23 March 2020 as the NHS was preparing for an influx of COVID-19 patients. All hospitals needed to adapt, with many surgical staff being redeployed in order to support the medical teams. The redeployment of doctors to the intensive treatment unit (ITU) and medical wards resulted in a

Authors: ${ }^{\text {A }}$ physician associate, QEQM Hospital, Margate, UK; ${ }^{\text {B }}$ core surgical trainee, QEQM Hospital, Margate, UK; ' locum orthopaedic consultant, QEQM Hospital, Margate, UK; ${ }^{\mathrm{D}}$ consultant orthopaedic surgeon, QEQM Hospital, Margate, UK lack of junior cover for surgical wards and the on-call team. In our institution, there are a number of physician associates (PAs) working within the orthopaedic department, reflecting an increase in the number of PAs trained in the UK over the last 10 years. 1 The

PA role was first developed in the USA and the profession has since expanded across a number of different countries. ${ }^{2}$ The university local to this institution has been training PAs since 2016.

PAs are postgraduates trained in the medical model with an emphasis on general medicine. ${ }^{3}$ They complete a 2-year programme with didactic and clinical teaching including placements in general practice, general medicine and in a variety of medical and surgical specialties. ${ }^{4}$ To qualify, they must also pass the UK PA national exam which encompasses 300 multiple choice questions (MCQ) and an 18-station observed structured clinical examination (OSCE). In order to maintain their managed voluntary registration, PAs must pass the MCQ portion of the examination every 6 years. ${ }^{5}$ This maintains the PAs broad medical knowledge throughout their career, making them a flexible workforce able to adapt in different ways dependent on departmental needs.

PAs are able to clinically assess patients, formulate management plans and instigate treatment (under the supervision of their consultant or general practitioner). A recent mixed-methods study over six acute care hospitals in the UK found that PAs working across medical and surgical specialties were 'acceptable, appropriate and safe' members of the teams. They were reported to contribute positively to continuity, patient experience and flow as well as aiding the induction of new doctors. They are also able to support the workload, releasing doctors to attend more complex patients or for training purposes. ${ }^{6}$ A systematic review of research on the impact of PAs in secondary care, including trauma and orthopaedics, found that there was little or no negative effect on health outcomes or cost when the PA/medical doctor (MD) model was compared with the MD-only model of care. ${ }^{7}$

Queen Elizabeth The Queen Mother (QEQM) Hospital, Margate, in East Kent is a district general hospital that has a busy trauma and orthopaedics (T\&O) department. This team is staffed by 13 consultants, 10 registrars, seven senior house officers ( $\mathrm{SHOs}$ ) and one PA covering days, two night resident medical officers (RMOs) with another PA on maternity leave. The PA working in the department had been qualified and worked in T\&O for 6 months, another newly qualified PA, who had been working in general surgery for 6 months was due to start in the department, started on 06 April 2020 and one PA returned from maternity leave on 20 April 2020. 
Table 1. The available workforce for the orthopaedic department pre- and during the COVID-19 pandemic

\begin{tabular}{lll} 
Role & Pre-COVID & COVID \\
Consultant & 13 & 9 \\
Registrar & 10 & 8 \\
Day SHO & 7 & 1 \\
Day PA $^{a}$ & 1 & 3 \\
Night RMO & 2 & 2 \\
\hline
\end{tabular}

${ }^{a}$ ward-based staff. $\mathrm{PA}=$ physician associate; $\mathrm{RMO}=$ resident medical officer; $\mathrm{SHO}=$ senior house officer.

Prior to the COVID-19 pandemic, the T\&O department worked across a trauma and a separate elective ward. The day-to-day care of the patients is managed by SHO grade doctors, supported by the aforementioned PAs. The PAs' role in the department was to work with the surgical team to support the care of inpatients (trauma and elective), as well as assessing patients in the outpatient setting and assisting in theatre. More specifically, this would include clinical review of new and on-going patients on ward rounds and actioning jobs identified across all orthopaedic teams. There was also an administrative role of completing admission and discharge documentation, as is also expected of $\mathrm{SHO}$ grade doctors. The PA role was chosen to bolster deficits within the trauma and orthopaedic department because of their generalist medical model of training.

The COVID-19 outbreak necessitated a restructuring of the department in order to adapt to the loss of staff through redeployment and illness. The majority of the junior medical staff working in T\&O were redeployed to the medical wards. Having PAs already working in surgery, we felt these gaps could be filled by increasing PA staffing. Therefore, PAs working across surgery were redeployed to T\&O. An additional senior PA returned to the department, producing a team of three PAs (Table 1). A new PA/MD team was responsible for the care of all T\&O inpatients.

The staffing alterations were simultaneously implemented with the cancellation of elective surgery and the reorganisation of the wards in order to accommodate the extra medical patients. Prior to the re-organisation of the department, there were 22 elective surgery inpatient beds and 30 trauma beds. With elective surgery cancelled, the inpatient number was significantly reduced, at the same time, an aggressive discharge policy was implemented. This meant that previous obstacles to discharge (such as social circumstances) were remedied more quickly than prior to lockdown, ensuring there was very little delay to discharge. PAs were an integral part of this. Immediately after lockdown was implemented due to the aforementioned reasons, there was a smaller volume of all trauma patients. However, the number of trauma patients and acuity increased to pre-COVID levels rapidly as people's behaviour returned to 'normal' in combination with the saturation of community settings, reducing the capability to discharge patients quickly.

During this period, the named consultant team structure gave way to a new unified team of PAs with an $\mathrm{SHO}$ working together. This team provided care for all the T\&O inpatients. The need for this was mainly due to redeployment of SHOs, which meant a more prominent role within the team for PAs. This team was able to share this as well as the on-call workload on a daily basis, providing 7-day cover for over 6 weeks. Here, we investigate whether this cover was adequate in these unique circumstances. Our hypothesis was that, using the PA/MD model, we were able to meet the standards of the (almost exclusively) MD-only model prior to the COVID-19 pandemic and used throughout the pandemic by the general surgical team.

\section{Materials and methods}

The study employed a combination of qualitative and quantitative data in order to investigate whether the PA/MD model of care would maintain standards of care during the COVID-19 pandemic and redeployment of the ward junior doctors. All data were collected over April and May 2020.

Ward nursing staff and orthopaedic registrars received a questionnaire focused around four key domains of surgical inpatient care: patient safety, continuity of care, patient flow and medical support for the ward. The questionnaire asked teammembers to compare the care provided during the COVID-19 pandemic with that of pre-COVID conditions. They rated each domain as 'better', 'same' or 'worse', and were then asked to give comments on each domain. All questionnaires were anonymised as much as possible. The questions were as follows.

$>$ How is patient safety compared with pre-COVID conditions?

$>$ How is continuity of care compared with pre-COVID conditions?

$>$ How is patient flow compared with pre-COVID conditions?

$>$ How is medical ward support compared with pre-COVID conditions?

The study also made use of available data on venous thromboembolism (VTE) assessments as a qualitative measure of efficiency of ward work completion. This allowed a comparison to the general surgical team who persevered with the traditional MDonly model of care. These data were collected prospectively via the VitalPAC system which makes a record of when a VTE assessment has been completed for each individual patient and if this was done in a timely manner. This is a responsibility that lies with the junior medical staff and must be done as soon as possible following admission to hospital as per National Institute for Health and Care Excellence (NICE) quality standard [QS3]. ${ }^{8}$ We also prospectively measured the average time to completion of discharge summaries and to-take-out (TTO) medication scripts. To measure this, a note of medically fit patients awaiting discharge was made. The electronic discharge notification (EDN) system was then used to assess how many of these patients had their discharge paperwork completed. This was measured daily over a 3-week period between 29 April 2020 and 19 May 2020 during the junior doctor redeployment.

\section{Results}

There were a total of 15 responses to the questionnaire. Six registrars and nine nurses responded, including staff nurses, ward managers / senior sisters and a nursing manager. The questionnaires were completed by substantive members of staff working in the department before and during the COVID-19 pandemic.

Patient safety, continuity of care, patient flow and medical ward support were all felt to be either better than pre-COVID (64\%) or the same as pre-COVID (36\%) working conditions. No responders felt that care was worse. 


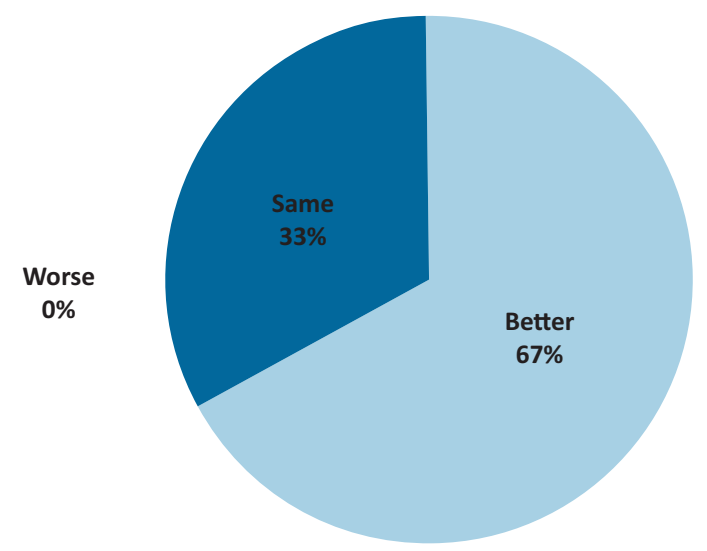

Fig 1. Perception of patient safety compared with pre-COVID-19 conditions.

\section{Patient safety}

Patient safety was considered by all respondents to be non-inferior to pre-COVID medical staffing for orthopaedic inpatients; 10/15 $(67 \%)$ respondents felt that patient safety was better than before, while 5/15 (33\%) felt it was the same (Fig 1).

Comments:

Ward nurse: Someone always on ward to go to with concerns.

Ward nurse: Patients are reviewed quicker.

Ward nurse: Things are being followed through.

Nurse manager: Had patients been split over four or five clinical areas then there would have been a significant risk were a patient to deteriorate, as they were over two wards, then there was always somebody nearby to assist with these patients.

Registrar: The combination of RMO and PA was excellent in ensuring patient safety.

\section{Continuity of care}

Continuity of care was judged by all respondents to be non-inferior to pre-COVID conditions, with $73 \%$ of respondents reporting an improvement (Fig 2).

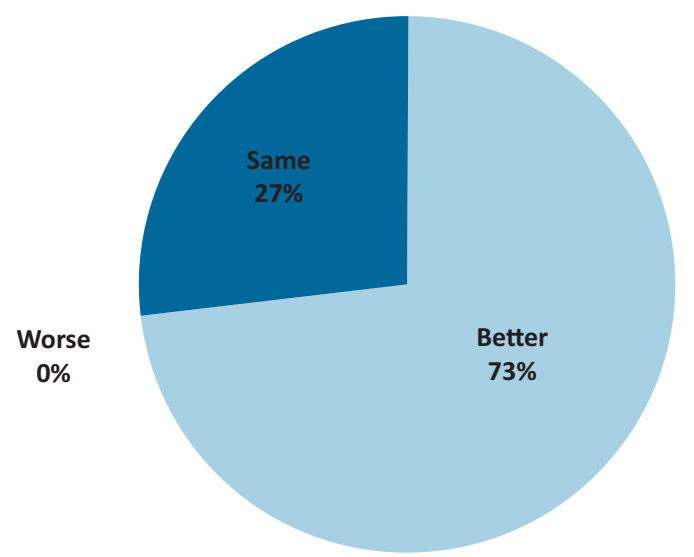

Fig 2. Perception of continuity of care compared with pre-COVID-19 conditions.

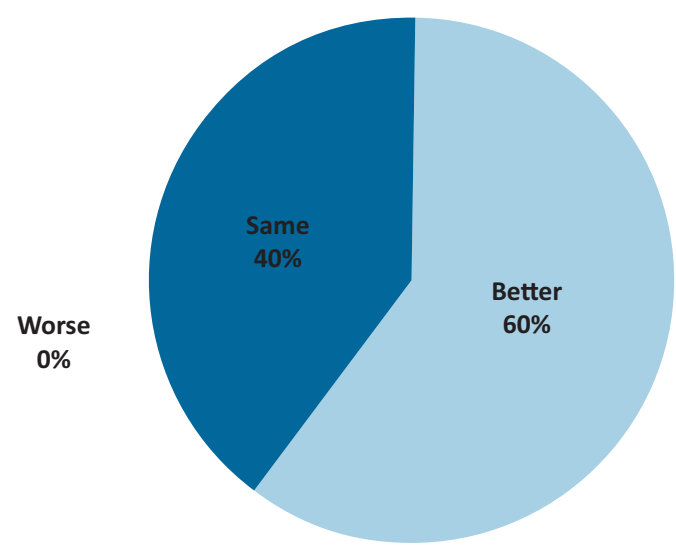

Fig 3. Perception of patient flow compared with pre-COVID-19 conditions.

\section{Comments:}

Ward nurse: PAs get tasks started sooner, more visual.

Ward nurse: Same staff on the wards provide better continuity. Ward nurse: Doctors are more available.

Nurse manager: There was a clear presence of T\&O surgeons or registrars on ward rounds with clear plans for those patients. There has also been consistent and visible presence from the PA team.

Registrar: We managed to maintain continuity of care but also had fewer overall patients being admitted.

Registrar: Continuity was improved with the SHO/PA model who know the patients well.

\section{Patient flow}

The results for patient flow also showed that conditions had not deteriorated, with $60 \%$ reporting improvement and $40 \%$ indicating things were the same (Fig 3).

\section{Comments:}

Ward nurse: Better because medical staff [are] more available. Ward nurse: Easier to get patients discharged.

Ward nurse: EDNs are being done more efficiently than before. Ward nurse: [The] situation is different because normally the patients are spread across four floors, not two.

Nurse manager: I think some of the processes put in place, such as board rounds, limited the impact of removing juniors from the team, I am not sure if there would have been a significant risk to patient safety or flow otherwise.

Registrar: The combination of RMO and PA was excellent as they know the patients well. This helps facilitate discharge planning.

\section{Medical ward support}

Sixty per cent of respondents felt that medical ward support was better compared with pre-COVID conditions, while $40 \%$ felt it was the same (Fig 4).

Comments:

Ward nurse: [It's] easier to get hold of someone than previously. Ward nurse: Never felt not supported. 


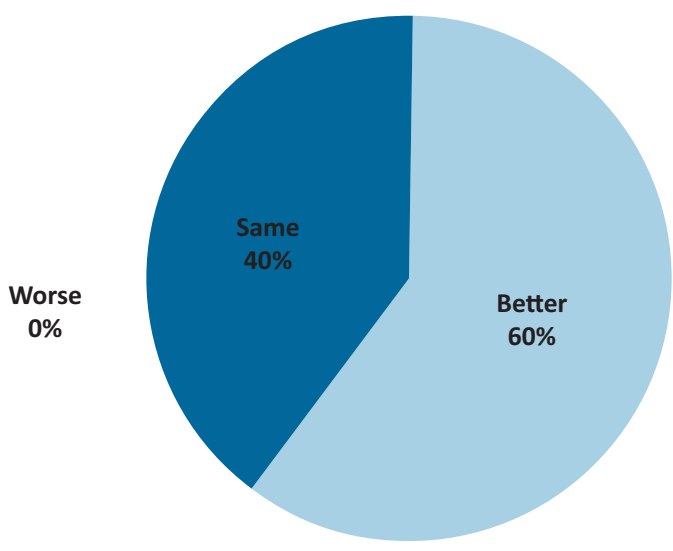

Fig 4. Perception of medical ward support compared with pre-COVID-19 conditions.

Ward nurse: Medical staff are more visible but need to be on the ward more.

Ward nurse: Greater availability of team mean quick decisions and appropriate plans can be made, especially at daily board rounds.

Nurse manager: I cannot recall any issues that have arisen as a result of the PAs covering the day-to-day duties on the ward, although the ordering of certain tests such as COVID-19 swabs in the first few weeks was prolonged due to requesting rights.

Nurse manager: As with all roles, they require senior engagement in order to function to the best of their abilities. I think that having observed how other teams have performed in the same period, T\&O has excelled.

Registrar: All team members were working together in harmony. Registrar: Smooth and effective handovers.

\section{VTE assessment}

We looked at the 3 months leading up to the COVID-19 redeployment in order to see how the department was performing up to this date. We then compared this with the month of April, when the junior doctors had been redeployed. The average compliance for orthopaedics fell by $3.7 \%$ in April, whereas this compliance for general surgery fell by $11.9 \%$ (Fig 5).

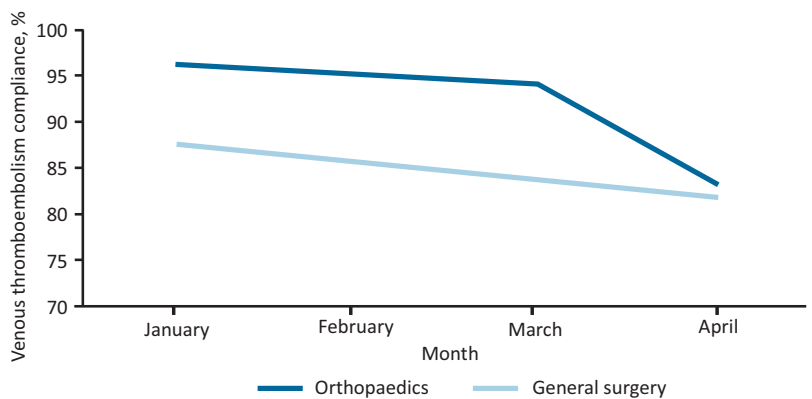

Fig 5. Venous thromboembolism assessment compliance for the orthopaedic and general surgical teams leading up to and during the COVID-19 junior doctor redeployment in April.

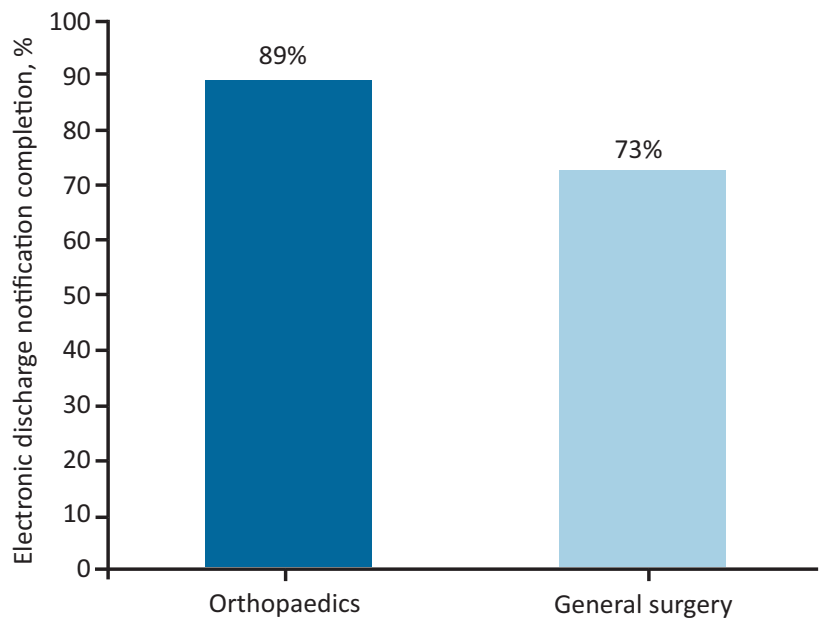

Fig 6. The average electronic discharge notification completion rate for the orthopaedic and general surgical teams over a 3-week period during the junior doctor redeployment.

\section{Electronic discharge efficiency}

The average number of orthopaedic and general surgery patients on the ward was 15 and 13 per week, respectively. When analysing the patients deemed to be medically fit awaiting discharge, the compliance with EDN completion was $89 \%$ for the orthopaedic team and $73 \%$ for the general surgical team (Fig 6).

\section{Discussion}

All respondents felt patient safety standards were maintained to the same level as before the redeployment and two-thirds felt that there had in fact been an improvement. The results of the VTE compliance audit show that the performance of the general surgical team was impacted by the COVID-19 redeployment to a greater extent ( $-11.9 \%$ compared with $-3.7 \%)$. During the redeployment of doctors, PAs were able to carry on with the day-to-day tasks on the ward including VTE assessments and completing EDNs, whereas the general surgical team, relying on more senior medical staff, were unable to keep up with these essential tasks. The results show the impact of PAs in mitigating the risk to patient safety and maintaining pre-COVID standards. These results are in keeping with published literature. ${ }^{6,9,10}$

With the changes put in place during the COVID-19 pandemic, there was an even greater emphasis on maintaining patient flow. A reduced number of surgical beds and measures in place within the community meant expedient discharges were necessary and possible. The PA model proves to be an excellent facilitator of patient flow, as demonstrated by this study. The availability of the PAs on the ward meant that jobs such as EDNs were completed quickly. We hypothesised that if only registrars and consultants were available to complete ward tasks, they are not done as efficiently because these staff members are not as familiar with the systems in place and have other responsibilities. We showed this with the data gathered around EDN completion and VTE assessment and comparing it with the general surgical team who, during the COVID-19 pandemic, relied upon registrars and consultants. Despite having greater patient numbers, the orthopaedic team was able to maintain better rates of EDN 
completion. The PA is in an optimal position to support early senior decision making as they are more likely to work in a department for longer thus gaining close working relationships with the senior doctors and systems knowledge relevant to each department and hospital. The PAs working in this institution at this time had all worked in the hospital for at least 6 months and were familiar with the computer systems and most referral pathways as well as already having established good relationships with the registrars and consultants in the department. Junior doctors passing through departments on rotation take time to acquire this and must re-learn on each rotation. ${ }^{6,9,11}$

Delivering safe and effective healthcare is complex due to the variety of patient needs and the large multidisciplinary team required. It is therefore difficult to measure the impact made by one component in particular. The strength of this study was in combining subjective evidence in the form of a questionnaire with objective evidence from available datasets. The combination of quantitative and qualitative evidence paints an accurate picture of the impact of the change in the medical workforce in our department.

The results of the questionnaire demonstrate that the ward was well supported by the orthopaedic medical team during the crisis. However, we know there are some important issues with this model of care. Due to lack of regulating body, PAs are unable to prescribe medication or order ionising radiation. This limits the role of the PA in UK practice and necessitated the presence of a medically qualified professional to carry out these tasks. As the role develops and expands, it is expected that the necessary legislation will be put in place, as it has been in the USA and the Netherlands. ${ }^{11,12}$ Following public consultation in 2018, the General Medical Council agreed to regulate PAs, a move likely to lead to the legislation required to give them prescribing rights and the right to order ionising radiation. ${ }^{13}$ Part of the success of our staffing redeployment can be attributed to the team's ability to work together while bringing the PA role to more prominence, and this is a cornerstone of other units wishing to pursue this model of care.

\section{Conclusion}

Overall, the three components of the study support the hypothesis that a PA/MD model of care is non-inferior to a MD-only model of care and was effective in withstanding the pressures of the COVID-19 pandemic junior doctor redeployment.

\section{References}

1 Aiello M, Roberts KA. Development of the United Kingdom physician associate profession. J Am Acad Physician Assist 2017;30:1-8.

2 Ballweg R, Rick TJ. Physician assistants and the expanding global health-care workforce. Am J Trop Med Hyg 2017;97:643-4.

3 Faculty of Physician Associates. Who are physician associates? Royal College of Physicians. www.fparcp.co.uk/about-fpa/Who-arephysician-associates [Accessed 30 May2020].

4 Department of Health, NHS National Practitioner Programme. The competence and curriculum framework for the physician assistant. Department of Health, 2006. https://bulger.co.uk/prison/ Physician \% 20Assistant.pdf [Accessed 30 May 2020].

5 Faculty of Physician Associates. Examinations. Royal College of Physicians. www.fparcp.co.uk/examinations [Accessed 30 May2020].

6 Drennan VM, Halter M, Wheeler C et al. What is the contribution of physician associates in hospital care in England? A mixed methods, multiple case study. BMJ Open 2019;9:e027012.

7 Halter $\mathrm{M}$, Wheeler $\mathrm{C}$, Pelone $\mathrm{F}$ et al. Contribution of physician assistants/associates to secondary care: a systematic review. BM] Open 2018;8:e019573.

8 National Institute for Health and Care Excellence. Venous thromboembolism in adults: reducing the risk in hospital: Quality standard [QS3]. NICE, 2018. www.nice.org.uk/Guidance/QS3 [Accessed 30 May 2020].

9 Timmermans MJC, van Vught AJAH, Peters YAS et al. The impact of the implementation of physician assistants in inpatient care: A multicenter matched-controlled study. PLoS One 2017;12:e0178212.

10 Timmermans MJC, van den Brink GT, van Vught AJAH et al. The involvement of physician assistants in inpatient care in hospitals in the Netherlands: a cost-effectiveness analysis. BMJ Open 2017;7:e016405.

11 Cawley JF, Hooker RS. Physician assistants in American medicine: the half-century mark. Am J Manag Care 2013;19:e333-41.

12 Nederlandse Associatie Physician Assistants. English information. NAPA. www.napa.nl/english.

13 Department of Health and Social Care. The regulation of medical associate professionals in the UK. DHSC, 2019. https://assets. publishing.service.gov.uk/government/uploads/system/uploads/ attachment_data/file/777130/maps-consultation-report.pdf [Accessed 30 May 2020].

Address for correspondence: Roz Tucker, Queen Elizabeth The Queen Mother Hospital, St Peter's Road, Margate, Kent CT9 4AN, UK.

Email: rosamund.tucker@nhs.net 